\section{Relationship between the optic nerve sheath diameter and lumbar cerebrospinal fluid pressure in patients with normal tension glaucoma}

A Pircher ${ }^{1}$, M Montali ${ }^{1,2}$, J Berberat ${ }^{2}$, L Remonda ${ }^{2}$ and HE Killer ${ }^{1}$

\begin{abstract}
Purpose To investigate on the relationship between the optic nerve sheath diameter (ONSD) and the lumbar cerebrospinal fluid pressure (CSF-p) in Caucasian patients with normal tension glaucoma (NTG).

Patients and methods Retrospective analysis of medical records of patients with open-angle glaucoma in the period from 2005 to 2015 from the Ophthalmology Department, Cantonal Hospital Aarau, Switzerland was performed. A total of 38 patients (mean age $68.6 \pm 11.3$ years, 21 females and 17 males) fulfilled the diagnostic criteria of NTG and underwent computed tomography (CT) of the orbit and lumbar puncture (LP). In total, 38 age- and gender-matched Caucasian subjects (mean age: $68.9 \pm 10.9$ years) without known ON diseases served as controls for ONSD measurements. ONSDs were measured at a distance of $3 \mathrm{~mm}$ from the posterior globe and lumbar CSF-p was related to the measurements. Statistical analysis was performed by using the independent two-tailed $t$-test and the nonparametric Spearman's correlation test. Results The mean ONSD in NTGs measured $6.4 \pm 0.9 \mathrm{~mm}$ and in controls $5.4 \pm 0.6 \mathrm{~mm}$. The difference between NTGs and controls showed statistical significance ( $t$-test: $P<0.000)$. The mean CSF-p in NTG was $11.6 \pm 3.7 \mathrm{~mm} \mathrm{Hg}$. There was no statistical significant correlation between ONSD and CSF-p (Spearman's correlation coefficient $\rho=0.06, P=0.72$ ).

Conclusions This study demonstrates enlarged ONSDs and normal lumbar CSF-p in 38 Caucasian NTG patients. As enlarged ONSDs generally are associated with increased intracranial CSF-p, these results can be explained by a disturbed communication
\end{abstract}

of CSF-p between the intracranial and intraorbital subarachnoid spaces.

Eye (2017) 31, 1365-1372; doi:10.1038/eye.2017.70; published online 28 April 2017

\section{Introduction}

Normal tension glaucoma (NTG) is a yet poorly understood disease of the optic nerve $(\mathrm{ON})$. It is characterized by visual field loss and optic disc excavation consistent with primary open-angle glaucoma despite a normal intraocular pressure (IOP). Vascular dysregulation ${ }^{1}$ and low cerebrospinal fluid pressure (CSF-p) are suggested to be possible contributing factors ${ }^{2,3}$ to the pathophysiology. Moreover, studies on CSF-p measured by lumbar puncture (LP) found a lower lumbar CSF-p in patients with primary open-angle glaucoma when compared to nonglaucomatous control subjects ${ }^{2}$ and even more so in NTG versus high-tension glaucoma. ${ }^{3}$

Several clinical and experimental studies correlated the CSF-p and the diameter of the subarachnoid space (SAS) of the ON. The studies applied ultrasound (US), ${ }^{4}$ computed tomography $(\mathrm{CT}),{ }^{5}$ and magnetic resonance imaging (MRI). ${ }^{6}$ While increased CSF-p is associated with a dilatation of the ON-SAS, , , -9 patients with reduced CSF-p showed a diminished diameter. ${ }^{10}$ This close correlation is explained by the continuity of the CSF containing spaces, given an open pathway and a homogenous CSF flow from the spine to the brain and from there to the SAS of the ON.

With regard to these findings one would expect a smaller ONSD in NTG, as a consequence of a lower CSF-p. However, data in the literature ${ }^{11,12}$ are contradictive and the ONSD in NTG remains still a matter of debate as
${ }^{1}$ Department of Ophthalmology, Cantonal Hospital Aarau, Aarau, Switzerland

${ }^{2}$ Department of Neuroradiology, Cantonal Hospital Aarau, Aarau, Switzerland

Correspondence: HE Killer, Kantonsspital Aarau Tellstrasse, CH-5001 Aarau, Switzerland Tel: +41 (0) 6283850 03; Fax: +41 (0) 628385005. E-mail: killer@ksa.ch

Received: 22 September 2016

Accepted in revised form: 17 March 2017 Published online: 28 April 2017 
the CSF-pathway, particularly in NTG cannot necessarily be considered as patent and CSF-p is not evenly distributed within all central nervous system (CNS) spaces. $^{13-15}$

In the present study, we measured the ONSD in 38 Caucasian patients with NTG and compared them to an age- and gender-matched control group of 38 Caucasian subjects without known ON diseases. In the NTG group we additionally examined the lumbar CSF-p and correlated the lumbar CSF-p to the ONSD.

\section{Subjects and methods}

This study was approved by the local ethical commission and followed the tenets of the Declaration of Helsinki.

A total of 753 medical records of patients with primary open-angle glaucoma from the Glaucoma Division of the Ophthalmology Department, Cantonal Hospital Aarau, Switzerland in the period from 2005 to 2015 were retrospectively analyzed. Of these, a total of 56 patients (99 of 112 eyes) fulfilled the criteria for NTG and underwent $\mathrm{CT}$ of the orbits combined with cisternography. In 38 (21 females and 17 males) of 56 patients additionally LP was performed at the beginning of the CT-cisternography and included in the current study. CT cisternography was performed if visual field defects showed progression despite a low IOP. Only one eye (randomly selected) of each patient was used for the analysis. The informed consent from the subjects was checked before including in this study.

NTG was diagnosed on the base of glaucomatous optic disc cupping on ophthalmoscopy and concomitant visual field defects. IOPmax (untreated and treated) was always $<21 \mathrm{~mm} \mathrm{Hg}$ and the visual field mean deviation was $\geq 3 \mathrm{~dB}$ shown by using standard automated perimetry (Program G2, Octopus Haag-Streit, Switzerland) at time of LP. Ophthalmoscopy, interpretation of the ON head and standard automated perimetry was performed by two experienced glaucoma specialists (HEK and GJ).

Each patient underwent full ophthalmologic examination including slit lamp assisted biomicroscopy, applanation tonometry, gonioscopy, measurement of central corneal thickness, standard automated perimetry, and neuroretinal rim assessment by using OCT (Heidelberg engineering, CA, USA). For each patient, IOP was measured at least four times at different times during the day (between 08:00 and 20:00 hours) in a seated position, using Goldmann applanation tonometry and twice at night (between 09:00 and 18:00 hours) in a recumbent position, using Perkins tonometry. The mean of all six IOP measurements performed during the same day as LP and CT cisternography were obtained, were taken for the analysis. All IOP measurements were examined for its dependence on central corneal thickness in order to exclude false negative values. ${ }^{16}$ IOP lowering treatment consisted of topical applied prostaglandin analogs, b-blockers, carbonic anhydrase, a-agonists, and combinations of these medications. None of the patients was on a medication that could influence the production or resorption of CSF.

\section{Controls}

From the database of the Department of Neuroradiology, Cantonal Hospital Aarau, Switzerland 38 age- and gender-matched Caucasian individuals without known intracranial or ON diseases who underwent CT of the cranium were recruited in a retrospective manner and served as controls.

All patients who underwent CT of the cranium as part of work-up for suspected cerebral stroke in the period from 2010 to 2015 were used as the initial data set. In total, 38 age- and gender-matched patients without documented intracranial or $\mathrm{ON}$ diseases and without head trauma or fractions of the skull and face were included. Only one eye (randomly selected) of each patient was included. None of the controls were taking carbonic anhydrase inhibitors. Exclusion and inclusion criteria were verified in the medical records and by a personal conversation via phone in most of the controls.

LP was performed only in the NTG group at the beginning of CT-cisternography. The CSF-opening pressure was measured for diagnostic reasons as lumbar CSF-p was demonstrated to be lower in primary openangle glaucoma. ${ }^{2}$ CSF-p measurements were not carried out due to any other pathology. LP was performed on the same day when IOP measurements where undertaken. It was performed at the same institution and by the same neuro-radiologist (LR) in order to provide the most homogenous examination as possible in all patients. LP was performed in a decubitus position while the initial opening pressure was obtained in the lateral recumbent position. The patient's legs were straightened and the patient was asked to remain calm and not to speak during the measurements to avoid a Valsalva manoeuver. The time between lumbar CSF-p measurement and CT was $15 \mathrm{~min}$ and was the same in all patients.

During LP $10 \mathrm{ml}$ of CSF was sampled for chemical analysis and then $10 \mathrm{ml}$ iopamidol was injected intrathecally. The total CSF volume was therefore not altered. The sampling of CSF was used for quantification of beta-trace protein and analyzed in a not yet published work. The patient was then turned to the prone position.

CT was performed in both NTG and controls. A 64-detector scanner (Aquillion 64, Toshiba, Tokyo, Japan) providing $0.5 \mathrm{mmX32}$ section collimation was used. Scanning parameters were a $25 \mathrm{~cm}$ field of view with a $512 \times 512$ matrix, and a soft tissue and a bone 
reconstruction algorithm were employed. The field of view included the foramen magnum and the nose.

Multiplanar reconstruction images were obtained in the axial, coronal, and sagittal planes with a $0.5 \mathrm{~mm}$ slice thickness. CT images were analyzed using the program VitreaCore (Vital Images, Inc., Minnetonka, MN, USA) on the Advantage Workstation 4.1 software (General Electric, Milwaukee, WI, USA).

For measuring the ONSDs (ON plus SAS plus sheaths), axial, sagittal, and coronal sections were used. All diameters were measured at $3 \mathrm{~mm}$ distance from the posterior globe.

In the axial section, the horizontal diameter was measured after formatting the images into an axial plane perpendicular to the $\mathrm{ON}$, while in the sagittal section the vertical diameter was measured after formatting the images into a sagittal plane perpendicular to the ON. Measurements in the coronal section verified both the horizontal and vertical diameter of the ONS, measured in the axial and sagittal sections (Figure 1). The average diameter of the ONS was calculated as the mean of the horizontal (axial and coronal sections) and vertical (sagittal and coronal section) diameter. All ONSD measurements were reviewed by an experienced neuroradiologist blinded to the ophthalmological examination.

In total, 7 of the 38 patients underwent additionally to CT scans MRI of the orbits (3 T Skyra whole body magnet, Siemens Healthcare, Erlangen, Switzerland) using a 32-channel head coil. Measurements were done in 3 dimensional T2 space sequence (acquisition time 05:05 min, repetition time $1400 \mathrm{~ms}$, echo time $139 \mathrm{~ms}$, echo train length 63 , flip angle 120 , matrix $256 \times 256$, FOV $150 \times 150,2$ averages). MRI was performed as part of a not yet published MRI study. Measurements on MRI images were performed to compare and confirm at least in seven patients the measurements done on CT images.
Statistical analysis was performed using SPSS 21.0 (IBM SPSS Inc., Chicago, IL, USA) for Windows statistical package. For analysis of differences between NTG patients and controls, male and female the independent two-tailed $t$-test for unpaired samples was used. For analysis of correlations between ONSD and CSF-p, ONSD and age, ONSD and IOP, and between CSF-p and age the non-parametric Spearman's rank order correlation coefficient was used.

\section{Results}

A total of 38 patients (mean age $68.6 \pm 11.3$ years, 21 females $(71.1 \pm 9.8$ years) and 17 males $(65.7 \pm 12.6$ years $)$, 67 of 76 eyes) fulfilled the criteria for NTG and had undergone CT of the orbit and LP. Only one randomly selected eye of each patient (38 eyes) were included and analyzed in the current study (Table 1). Mean glaucomatous visual field defect (mean deviation; $n=38$ ) was $13.4 \pm 8.1 \mathrm{~dB}$ and the mean IOP was $14.9 \pm 2.6 \mathrm{~mm} \mathrm{Hg}$.

The control group consists of 38 age- and gendermatched subjects without known $\mathrm{ON}$ diseases: corresponding to the NTG group only one randomly selected eye of each patient (21 eyes in females and 17 eyes in males) were included. Mean age was $68.9 \pm 10.9$ years, $71.4 \pm 9.7$ in females and $65.9 \pm 10.8$ in males (Table 2).

Subjects in the NTG and control group showed no statistically significant difference in either gender or age $(P=0.902)$.

The mean ONSD $(n=38)$ using CT in all NTG patients measured $6.4 \pm 0.9 \mathrm{~mm}$. It ranged from 5.4 to $9.1 \mathrm{~mm}$ $(6.3 \pm 0.9)$ in females $(n=21)$ and 5.0 to $8.0 \mathrm{~mm}(6.5 \pm 0.8)$ in males ( $n=17$; Table 1 and 2). There were no significant difference in ONSDs between females and males $(P=0.58)$. No significant correlation was shown between

\section{a}

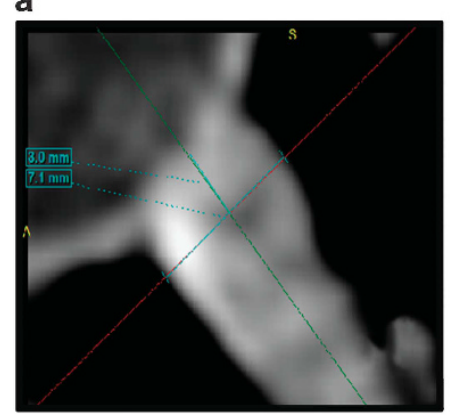

b

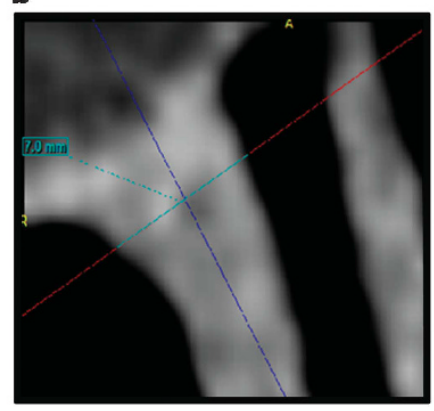

c

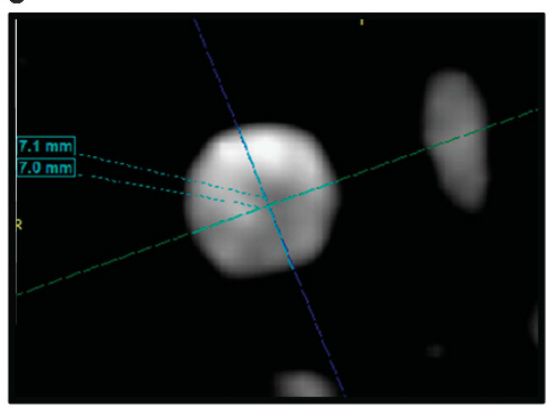

Figure 1 Measurement of the optic nerve sheath diameter (ONSD; $\mathrm{mm}$ ) of the left optic nerve $(\mathrm{ON})$ at $3 \mathrm{~mm}$ distance from the lamina cribrosa. Sagittal (a), axial (b), and coronal (c) sections were used. Note the distension in the retrobulbar ON portion: $7.0 \mathrm{~mm}$ in the vertical diameter and $7.1 \mathrm{~mm}$ in the horizontal diameter. 
Table 1 Measurements of IOP, lumbar CSF-p, and ONSD in patients with NTG

\begin{tabular}{|c|c|c|c|c|c|}
\hline \multirow[t]{2}{*}{$n$} & \multicolumn{5}{|c|}{ NTG-patients } \\
\hline & Age (m) & Age $(f)$ & $\begin{array}{c}I O P \\
(m m H g)\end{array}$ & $\begin{array}{c}\text { CSF-p } \\
(m m H g)\end{array}$ & $\begin{array}{c}\text { ONSD } \\
(\mathrm{mm})\end{array}$ \\
\hline 1 & 76 & & 16 & 5.9 & 5.8 \\
\hline 2 & 63 & & 15 & 7.4 & 7.4 \\
\hline 3 & 53 & & 17 & 7.4 & 6.4 \\
\hline 4 & 65 & & 10 & 11.8 & 5.9 \\
\hline 5 & & 66 & 12 & 13.9 & 5.4 \\
\hline 6 & & 58 & 14 & 8.1 & 6.8 \\
\hline 7 & 69 & & 15 & 22.1 & 7.5 \\
\hline 8 & & 64 & 14 & 8.1 & 9.1 \\
\hline 9 & 85 & & 13 & 11.8 & 6.4 \\
\hline 10 & & 75 & 17 & 16.2 & 6.9 \\
\hline 11 & 78 & & 16 & 12.5 & 5.0 \\
\hline 12 & 50 & & 18 & 15.5 & 6.1 \\
\hline 13 & & 60 & 16 & 8.8 & 7.2 \\
\hline 14 & & 72 & 16 & 18.4 & 7.4 \\
\hline 15 & & 68 & 13 & 16.2 & 5.4 \\
\hline 16 & 61 & & 18 & 13.9 & 7.1 \\
\hline 17 & 88 & & 16 & 6.6 & 5.5 \\
\hline 18 & & 74 & 14 & 9.5 & 5.9 \\
\hline 19 & & 72 & 17 & 13.9 & 5.4 \\
\hline 20 & 79 & & 13 & 10.3 & 8.0 \\
\hline 21 & 59 & & 18 & 13.9 & 7.1 \\
\hline 22 & & 91 & 15 & 11.0 & 5.4 \\
\hline 23 & & 65 & 16 & 11.0 & 5.8 \\
\hline 24 & & 83 & 15 & 7.4 & 6.1 \\
\hline 25 & 45 & & 16 & 12.5 & 6.4 \\
\hline 26 & 51 & & 12 & 13.9 & 7.0 \\
\hline 27 & 60 & & 12 & 16.2 & 6.0 \\
\hline 28 & & 53 & 8 & 8.1 & 6.3 \\
\hline 29 & 73 & & 15 & 15.5 & 6.0 \\
\hline 30 & & 58 & 20 & 8.8 & 6.0 \\
\hline 31 & & 67 & 15 & 8.8 & 5.9 \\
\hline 32 & & 80 & 18 & 11.0 & 6.2 \\
\hline 33 & & 76 & 18 & 13.9 & 6.1 \\
\hline 34 & & 77 & 16 & 11.0 & 6.0 \\
\hline 35 & & 86 & 14 & 7.4 & 5.6 \\
\hline 36 & & 68 & 10 & 8.1 & 6.5 \\
\hline 37 & 61 & & 17 & 10.3 & 6.8 \\
\hline 38 & & 79 & 12 & 13.2 & 7.7 \\
\hline $\mathrm{AM} \pm \mathrm{SD}$ & $71.1 \pm 9.8$ & $65.7 \pm 12.6$ & $14.9 \pm 2.6$ & $11.6 \pm 3.7$ & $6.4 \pm 0.9$ \\
\hline
\end{tabular}

Abbreviations: Am, arithmetic mean; CSF-p, cerebrospinal fluid pressure; f, female; IOP, Intraocular pressure; LP, lumbar puncture; m, male; NTG, normal tension glaucoma; ONSD, optic nerve sheath diameter. ONSD was measured in $3 \mathrm{~mm}$ distance from the posterior globe in $\mathrm{mm}$ IOP (mmHg) was measured by applanation tonometry and CSF-p $(\mathrm{mmHg})$ by LP. Age for $\mathrm{m}$ and $\mathrm{f}$ in years.

Bold entries highlight the arithmetic mean values.

age and ONSDs (Spearman's correlation; $\rho=-0.29$, $P=0.08)$.

The mean ONSD $(n=7)$ applying MRI measured $6.5 \pm 0.7 \mathrm{~mm}$ (cf. CT: $6.4 \pm 0.7 \mathrm{~mm}$ ). There was no significant difference ( $t$-test: $P=0.791$ ) between the measurements performed with CT and those with MRI.

The overall $(n=38)$ mean CSF-p was $11.6 \pm 3.7 \mathrm{~mm} \mathrm{Hg}$ and ranged from 5.9 to $22.1 \mathrm{~mm} \mathrm{Hg}$. In females $(n=21)$ it ranged from 7.4 to $18.4 \mathrm{~mm} \mathrm{Hg}(11.1 \pm 3.3)$ and from 5.9 to $22.1 \mathrm{~mm} \mathrm{Hg}(12.1 \pm 4.2)$ in males $(n=17$; Table 1$)$.

Between males and females there was no statistical significant difference $(P=0.38)$. No statistical significant correlation was shown between CSF-p and age $(\rho=-0.1$, $P=0.54)$.

Performed bivariate analysis comparing ONSD with CSF-p showed no statistical significant correlation between ONSDs and CSF-p ( $\rho=0.06, P=0.72$; Figure 2). Further, there was no significant correlation between ONSDs and IOP $(\rho=-0.01, P=0.97)$.

The mean ONSD $(n=38)$ using CT in the control group measured $5.4 \pm 0.6 \mathrm{~mm}$. It ranged from 4.3 to $6.6 \mathrm{~mm}$ $(5.5 \pm 0.6)$ in females $(n=21)$ and 4.6 to $6.4 \mathrm{~mm}(5.4 \pm 0.6)$ in males ( $n=17$; Table 2$)$. There was no significant difference in ONSDs between females and males $(P$ $=0.350$ ). A slightly significant correlation was shown between age and ONSDs (Spearman's correlation; $\rho=0.37, P=0.022$ )

Between NTG patients and controls a statistically significant difference in ONSD was found ( $t$-test: $P<0.000$; Figure 3). The difference showed statistical significance in females and males (females: $P=0.002$; males: $P<0.000)$.

\section{Discussion}

The present study compares the lumbar CSF-p and the ONSD in 38 Caucasian NTG patients. This study demonstrates enlarged ONSDs with normal lumbar CSF-p. ${ }^{17}$ When we observe both measurements plotted on a graph the CSF-p and the ONSD seem independent from each other (Figure 2).

Several clinical and experimental studies using sonography, ${ }^{4} \mathrm{CT},{ }^{5}$ or $\mathrm{MRI}^{6,7,18}$ reported a close correlation between CSF-p and the width of the ON-SAS. This correlation has been described between CSF-p measurements performed in different CSF spaces, ${ }^{7}$ including the SAS of the lumbar spine by using LP. ${ }^{18}$ Moreover, in a MRI study ${ }^{7}$ with 12 patients with subdural fluid collection this correlation has been found as a sigmoidal function. A study with 72 Han Chinese patients with different neurological disorders using MRI suggested a linear correlation within a certain CSF-p range. $^{18}$

Given an open CSF pathway through all CSF containing spaces, a close relationship between CSF-p and ONSD is to be expected. However, in studies applying cisternography, CSF appears to be segregated in a cohort of NTG patients. ${ }^{13}$ In addition, LPGDS (lipocalin-like prostaglandin D-synthase), a protein expected to be distributed homogenously in the entire CNS, was found to differ markedly between the lumbar CSF and the CSF surrounding the $\mathrm{ON}$ in a variety of $\mathrm{ON}$ diseases, 
Table 2 Measurement of the ONSD in patients with NTG and healthy controls

\begin{tabular}{|c|c|c|c|c|c|c|}
\hline \multirow[t]{2}{*}{$n$} & \multicolumn{3}{|c|}{ NTG patients } & \multicolumn{3}{|c|}{ Controls } \\
\hline & Age (m) & Age $(f)$ & ONSD $(\mathrm{mm})$ & Age (m) & Age $(f)$ & ONSD $(\mathrm{mm})$ \\
\hline 1 & 45 & & 6.4 & 48 & & 4.8 \\
\hline 2 & 50 & & 6.1 & 52 & & 4.7 \\
\hline 3 & 51 & & 7.0 & 53 & & 5.1 \\
\hline 4 & & 53 & 6.3 & & 53 & 5.3 \\
\hline 5 & 53 & & 6.4 & 54 & & 4.6 \\
\hline 6 & & 58 & 6.8 & & 57 & 5.3 \\
\hline 7 & & 58 & 6.0 & & 60 & 5.5 \\
\hline 8 & 59 & & 7.1 & 58 & & 5.6 \\
\hline 9 & 60 & & 6.0 & 58 & & 6.1 \\
\hline 10 & & 60 & 7.2 & & 61 & 4.5 \\
\hline 11 & 61 & & 6.8 & 61 & & 5.8 \\
\hline 12 & 61 & & 7.1 & 61 & & 5.3 \\
\hline 13 & 63 & & 7.4 & 65 & & 5.0 \\
\hline 14 & & 64 & 9.1 & & 65 & 5.3 \\
\hline 15 & 65 & & 5.9 & 65 & & 5.5 \\
\hline 16 & & 65 & 5.8 & & 66 & 6.0 \\
\hline 17 & & 66 & 5.4 & & 66 & 5.8 \\
\hline 18 & & 67 & 5.9 & & 67 & 5.8 \\
\hline 19 & & 68 & 5.4 & & 68 & 5.4 \\
\hline 20 & & 68 & 6.5 & & 69 & 5.9 \\
\hline 21 & 69 & & 7.5 & 67 & & 5.1 \\
\hline 22 & & 72 & 7.4 & & 72 & 5.6 \\
\hline 23 & & 72 & 5.4 & & 73 & 4.3 \\
\hline 24 & 73 & & 6.0 & 73 & & 5.4 \\
\hline 25 & & 74 & 5.9 & & 73 & 4.7 \\
\hline 26 & & 75 & 6.9 & & 76 & 6.6 \\
\hline 27 & 76 & & 5.8 & 77 & & 5.4 \\
\hline 28 & & 76 & 6.1 & & 78 & 6.0 \\
\hline 29 & & 77 & 6.0 & & 79 & 5.4 \\
\hline 30 & 78 & & 5.0 & 78 & & 4.7 \\
\hline 31 & & 79 & 7.7 & & 79 & 5.0 \\
\hline 32 & 79 & & 8.0 & 79 & & 6.4 \\
\hline 33 & & 80 & 6.2 & & 80 & 5.8 \\
\hline 34 & & 83 & 6.1 & & 83 & 5.8 \\
\hline 35 & 85 & & 6.4 & 83 & & 6.2 \\
\hline 36 & & 86 & 5.6 & & 83 & 6.4 \\
\hline 37 & 88 & & 5.5 & 88 & & 5.3 \\
\hline 38 & & 91 & 5.4 & & 92 & 5.6 \\
\hline $\mathrm{AM}_{ \pm} \mathrm{SD}$ & $65.7 \pm 12.6$ & $71.1 \pm 9.8$ & $6.4 \pm 0.9$ & $65.9 \pm 10.8$ & $71.4 \pm 9.7$ & $5.4 \pm 0.6$ \\
\hline
\end{tabular}

Abbreviations: Am, arithmetic mean; NTG, normal tension glaucoma; ONSD, optic nerve sheath diameter.

Measurement of the ONSD in $3 \mathrm{~mm}$ from the lamina cribrosa. Age for male $(\mathrm{m})$ and female (f) in years.

Bold entries highlight the arithmetic mean values.

indicating an inhomogeneous CSF composition. ${ }^{14}$ Although CSF-p between the lumbar spine and the cerebral parenchyma correlated in a study ${ }^{19}$ with ten patients with normal tension hydrocephalus, the authors point out that such a correlation is only possible 'given an open CSF pathway between the different CSF spaces'.

In the present study the ONSD in Caucasian NTG patients measured significantly $(P<0.000)$ larger diameters compared to age- and gender-matched Caucasian controls without $\mathrm{ON}$ diseases.

The findings of larger ONSDs in Caucasian NTG patients are in accordance with measurements in a study from Jaggi et al ${ }^{11}$ and contradictory to a study from
Wang et al ${ }^{12}$ that was performed with MRI in an exclusively Asian population. In contrast to these studies the measurements in the present cohort was performed in three dimensions (sagittal, axial, and coronar) in order to avoid oblique aberrations. Wang et al ${ }^{12}$ explained their results with a lower CSF-p. This argument, however, is based on data taken from an American ${ }^{2}$ population as well as from a study from $\mathrm{Asia}^{3}$ as they did not perform measurements of CSF-p in their cohort. They in fact combined data from different populations. Such differences in ONSDs are likely the result of different measuring techniques (CT vs MRI), individual sheath thickness, and elasticity between different races 


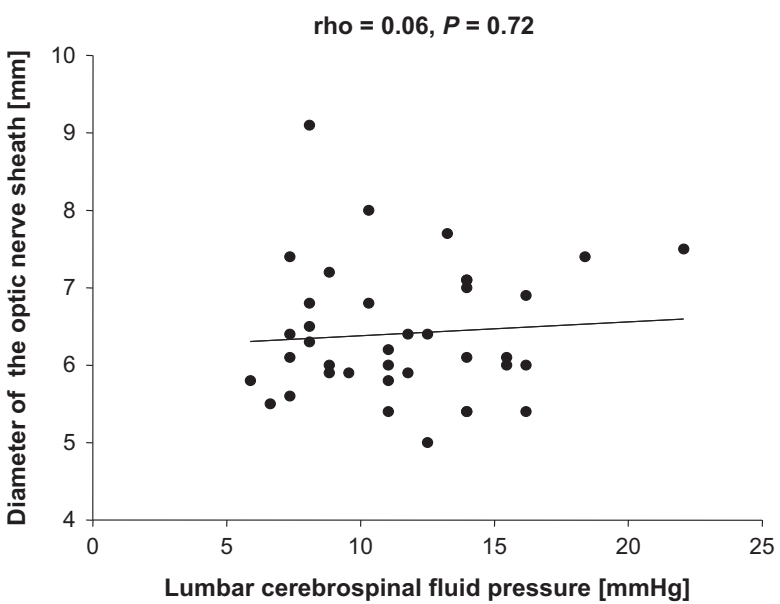

Figure 2 Scatter plot of the optic nerve sheath diameter (ONSD; $\mathrm{mm}$ ) and lumbar cerebrospinal fluid pressure (CSF-p; mmHg). Note the lack of relationship between the lumbar CSF-p and the ONSD in normal tension glaucoma (NTG) patients.

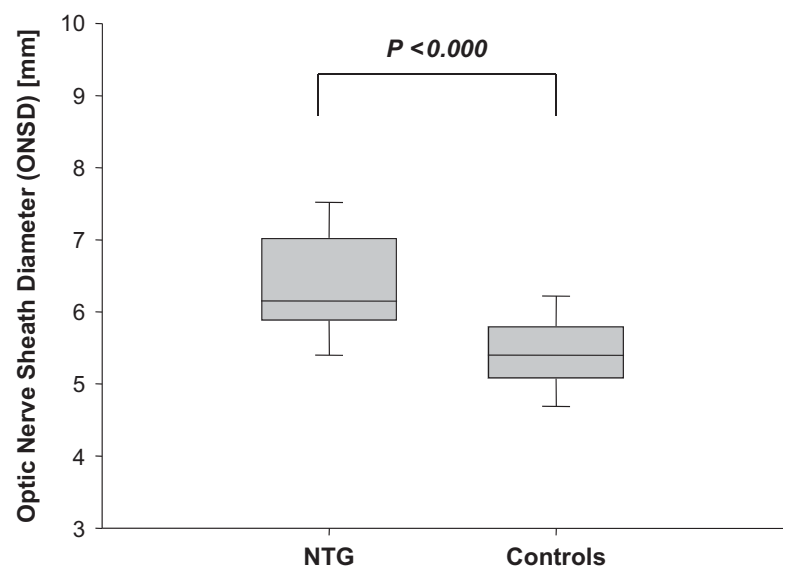

Figure 3 Box plot of optic nerve sheath diameter (ONSD) measurements in normal tension glaucoma (NTG) patients and controls. NTG patients $(n=38)$ and controls $(n=38)$. A statistically significant difference was found between NTG patients and controls.

(Caucasian vs Asian). However, the exact mechanism is still a matter of debate.

At least two mechanisms might explain enlarged ONSDs in NTG patients. First, a localized CSF-p elevation behind the globe due to impaired CSF outflow might lead to increased radial stress to the $\mathrm{ON}$ sheaths and thereby stretching the ON sheaths. Second, an accumulation of proteins of different biological functions. ${ }^{14}$ An accumulation of collagenases, ${ }^{20}$ for example, can effects the collagenous structure of the $\mathrm{ON}$ sheaths leading to a thinning of the dura mater similar to mechanisms prescribed in corneal thinning. ${ }^{21}$
The normal range of CSF-p in healthy is between 5 and $15 \mathrm{~mm} \mathrm{Hg}$ with a mean of $12 \mathrm{mmHg}$ when measured by LP. ${ }^{17}$ Lumbar CSF-p in our NTG patients $(n=38)$ measured $11.6 \mathrm{~mm} \mathrm{Hg}$. These measurements are in contrast to results from previous studies in NTG using invasive lumbar CSF-p measurement which found a lower lumbar CSF-p (Berdahl et al: ${ }^{22} 9.3 \pm 3.2 \mathrm{~mm} \mathrm{Hg}$ $(n=11)$, Ren et al: $\left.{ }^{3} 9.5 \pm 2.2 \mathrm{~mm} \mathrm{Hg}(n=14)\right)$. The discrepancy might at least in part be explained by differences in population. Our study group was a purely Caucasian population whereas in the study by Ren et $a l^{3}$ the examined group was an Asian population. The study from Berdahl et $a l^{22}$ was performed in the USA with no information about the population involved. A further explanation might be the rather small number of patients in their studies (Berdahl et al: ${ }^{22} n=11$, Ren et al: ${ }^{3} n=14$ ).

Along the ON, CSF flows between a complex system of chambers. ${ }^{23}$ Both the arachnoid and the pia mater that limit the CSF space are composted of meningothelial cells. Meningothelial cells are multifunctional cells that react with proliferation to a variety of biological and mechanical stimuli, for example, CSF-p elevation. ${ }^{24}$ Such proliferation can reduce the volume of the SAS thereby turning the SAS of the ON into a partially isolated CSF compartment with a most likely different CSF-p and different composition of proteins than present in other CSF spaces. ${ }^{25}$

Under the condition of a fixed relationship between CSF-p and ONS distension, the measurements in our study provide evidence that in NTG, the CSF-p at least in the SAS of the retrobulbar ON segment might differ from that in other CSF-spaces, for example, the lumbar spine SAS.

Similar to these findings in NTG, in 20 patients with idiopathic intracranial hypertension only $45 \%$ of the cases included in a MRI study, demonstrated a distension of the perioptic SAS despite an elevated lumbar CSF-p. ${ }^{6}$ Similar observations can be done in cases of asymmetric papilledema. This suggests that in other disorders of the ON such as NTG, the ON-SAS might not be patent and hence a close correlation between the lumbar CSF-p and the ONSD is questionable.

This study reports on results derived from a homogenous group of patients with NTG. The lack of a control group for lumbar CSF-p measurements is an obvious weakness. The question, however, is how would the proper control group look like? LP is not performed in healthy people for obvious ethical reasons and LP measurements in neurological patients are biased by their disease which might influence the CSF-p. Therefore we compared and discussed our lumbar CSF-p measurements with data from controls in other studies published in the literature. For further prospective studies 
it would be interesting to recruit patients who underwent non invasive CSF-p measurements. ${ }^{26}$

In summary, our data shows enlarged ONSDs and a normal intracranial CSF- $\mathrm{p}^{17}$ without a significant correlation between CSF-p and ONSD in 38 Caucasian patients with NTG. We suggest that a disturbed communication between the intracranial and intraorbital SAS might explain these findings.

\section{Summary}

\section{What was known before}

- In the last year, the role of cerebrospinal fluid (CSF) has gained interest in the pathophysiology of primary open angle glaucoma (POAG), particularly in normal tension glaucoma (NTG). Several studies found a lower lumbar CSF-p in patients with POAG when compared to nonglaucomatous control subjects and even more so in NTG versus high-tension glaucoma. The correlation between intracranial pressure and the diameter of the optic nerve sheath (ONSD) is an established method in the evaluation of papilledema. It has now found its way into evaluating cerebrospinal fluid pressure (CSF-p) in NTG. Study results, however, vary between distended ONSD and narrow ONSD.

\section{What this study adds}

- In this study we measured the lumbar CSF-p and the optic nerve sheath diameter (ONSD) and examined the correlation between them in patients with NTG. To the best of our knowledge there exists no study in the literature that compared the lumbar measured CSF-p with ONSDs performed in one and the same NTG population. The purpose of this study is to get a better understanding in CSF dynamics in NTG.

\section{Conflict of interest}

The authors declare no conflict of interest.

\section{Acknowledgements}

Special thanks to J Pircher, MD, Department of Cardiology, München, Germany for the technical support.

\section{References}

1 Flammer J, Orgül S, Costa VP, Orzalesi N, Krieglstein GK, Serra LM et al. The impact of ocular blood flow in glaucoma. Prog Retin Eye Res 2002; 21(4): 359-393.

2 Berdahl JP, Allingham RR, Johnson DH. Cerebrospinal fluid pressure is decreased in primary open-angle glaucoma. Ophthalmology 2008; 115(5): 763-768.

3 Ren R, Jonas JB, Tian G, Zhen Y, Ma K, Li S et al. Cerebrospinal fluid pressure in glaucoma: a prospective study. Ophthalmology 2010; 117(2): 259-266.
4 Hansen HC, KH, Kunze. K. Optic nerve sheath enlargement in acute intracranial hypertension. J Neuroophthalmol 1994; 14(6): 345-354

5 Zaidi SJ, Yamamoto LG. Optic nerve sheath diameter measurements by CT scan in ventriculoperitoneal shunt obstruction. Hawaii J Med Public Health 2014; 73(8): 251-255.

6 Brodsky MC, Vaphiades M. Magnetic resonance imaging in pseudotumor cerebri. Ophthalmology 1998; 105(9): 1686-1693.

7 Watanabe A, Kinouchi H, Horikoshi T, Uchida M, Ishigame $\mathrm{K}$. Effect of intracranial pressure on the diameter of the optic nerve sheath. J Neurosurg 2008; 109(2): 255-258.

8 Mader TH, Gibson CR, Pass AF, Kramer LA, Lee AG, Fogarty J et al. Optic disc edema, globe flattening, choroidal folds, and hyperopic shifts observed in astronauts after longduration space flight. Ophthalmology 2011; 118(10): 2058-2069.

9 Mader TH, Gibson CR, Pass AF, Lee AG, Killer HE, Hansen HC et al. Optic disc edema in an astronaut after repeat long-duration space flight. J Neuroophthalmol 2013; 33(3): 249-255.

10 Rohr A, Jensen U, Riedel C, van Baalen A, Fruehauf MC, Bartsch T et al. MR imaging of the optic nerve sheath in patients with craniospinal hypotension. AJNR Am J Neuroradiol 2010; 31(9): 1752-1757.

11 Jaggi GP, Miller NR, Flammer J, Weinreb RN, Remonda L, Killer HE. Optic nerve sheath diameter in normal-tension glaucoma patients. Br J Ophthalmol 2012; 96(1): 53-56.

12 Wang N, Xie X, Yang D, Xian J, Li Y, Ren R et al. Orbital cerebrospinal fluid space in glaucoma: the Beijing intracranial and intraocular pressure (iCOP) study. Ophthalmology 2012; 119(10): 2065-73. e1.

13 Killer HE, Miller NR, Flammer J, Meyer P, Weinreb RN, Remonda L et al. Cerebrospinal fluid exchange in the optic nerve in normal-tension glaucoma. Br J Ophthalmol 2012; 96(4): 544-548.

14 Killer HE, Jaggi GP, Flammer J, Miller NR, Huber AR. The optic nerve: a new window into cerebrospinal fluid composition? Brain 2006; 129(Pt 4): 1027-1030.

15 Mindermann T. Pressure gradients within the central nervous system. J Clin Neurosci 1999; 6(6): 464-466.

16 Shah S. Accurate intraocular pressure measurement-the myth of modern ophthalmology? Ophthalmology 2000; 107(10): 1805-1807.

17 Berdahl JP, Allingham RR. Intracranial pressure and glaucoma. Curr Opin Ophthalmol 2010; 21(2): 106-111.

18 Xie X, Zhang X, Fu J, Wang H, Jonas JB, Peng X et al. Noninvasive intracranial pressure estimation by orbital subarachnoid space measurement: the Beijing Intracranial and Intraocular Pressure (iCOP) study. Critical Care 2013; 17(4): R162.

19 Lenfeldt N, Koskinen LO, Bergenheim AT, Malm J, Eklund A. CSF pressure assessed by lumbar puncture agrees with intracranial pressure. Neurology 2007; 68(2): 155-158.

20 Golubnitschaja O, Yeghiazaryan K, Liu R, Mönkemann H, Leppert D, Schild $\mathrm{H}$ et al. Increased expression of matrix metalloproteinases in mononuclear blood cells of normaltension glaucoma patients. J Glaucoma 2004; 13(1): 66-72.

21 Balasubramanian SA, Pye DC, Willcox MD. Are proteinases the reason for keratoconus? Curr Eye Res 2010; 35(3): 185-191.

22 Berdahl JP, Fautsch MP, Stinnett SS, Allingham RR. Intracranial pressure in primary open angle glaucoma, normal tension glaucoma, and ocular hypertension: a casecontrol study. Invest Ophthalmol Vis Sci 2008; 49(12): 5412-5418. 
23 Killer HE, Laeng HR, Flammer J, Groscurth P. Architecture of arachnoid trabeculae, pillars, and septa in the subarachnoid space of the human optic nerve: anatomy and clinical considerations. Br J Ophthalmol 2003; 87(6): 777-781.

24 Xin X, Fan B, Flammer J, Miller NR, Jaggi GP, Killer HE et al. Meningothelial cells react to elevated pressure and oxidative stress. PloS One 2011; 6(5): e20142.
25 Killer HE, Subramanian PS. Compartmentalized cerebrospinal fluid. Int Ophthalmol Clin 2014; 54(1): 95-102.

26 Siaudvytyte L, Januleviciene I, Ragauskas A, Bartusis L, Siesky B, Harris A. Update in intracranial pressure evaluation methods and translaminar pressure gradient role in glaucoma. Acta Ophthalmol 2015; 93(1): 9-15. 